\title{
La enseñanza síncrona a distancia como método de formación continua a profesionales. La experiencia del "Curso Online de Estaciones de Bombeo"
}

\section{P. Amparo López Jiménez, Gonzalo López Patiño.}

Departamento de Ingeniería Hidráulica y Medio Ambiente, Universitat Politècnica de València. palopez@uov.es; glpatin@upv.es

\begin{abstract}
Any developed society must have an adequate system of continuous training of its professionals that guarantees their adaptation to technological and scientific progress. The education system itself is part of the mechanism of innovation. The potential of information technology and communication allow professionals to conduct training programs with more flexible schedules, without neglecting their jobs, without making expensive travel while maintaining the applicability of the training. This paper presents the development and experience of a training program for professionals synchronous distance engaged in design, operation and maintenance of pumping stations, as present in all types of industrial and agricultural activities. The program was attended by people from different countries, all working professionals, and has taught from different cities. To do this we used the media technicians and audiovisual classrooms Continuing Education Center of the UPV, for synchronous teaching, Policonecta teleteaching tool as online system, and polymorphous- $T$ as a system of information management training program.
\end{abstract}

\footnotetext{
Resumen

Cualquier sociedad desarrollada debe disponer de un adecuado sistema de formación continua de sus profesionales que les garantice su adaptación al progreso tecnológico y científico. El propio sistema de formación forma parte de ese mecanismo de innovación. Las posibilidades que ofrecen las tecnologías de la información y comunicación permiten que los profesionales puedan realizar programas de formación con horarios más flexibles, sin desatender sus puestos de trabajo, sin realizar costosos desplazamientos, pero manteniendo la aplicabilidad de la formación recibida. En este trabajo
} 
La enseñanza síncrona a distancia como método de formación continua a profesionales. La experiencia del "Curso Online de Estaciones de Bombeo"

se presenta el desarrollo, y la experiencia, de un programa de formación a distancia síncrona dirigido a profesionales que se dedican al diseño, operación y mantenimiento de estaciones de bombeo, tan presentes en todo tipo de actividades industriales y agrícolas. Al programa han asistido personas desde diferentes países, todos ellos profesionales en activo, y se ha impartido desde diferentes ciudades. Para ello se han utilizado los medios técnicos y personal de las aulas de audiovisuales del Centro de Formación Permanente de la UPV, para la docencia síncrona, la herramienta Policonecta como sistema de teledocencia en línea, y POLIFORMA-T como sistema de gestión de la información del programa de formación.

Palabras clave: Teledocencia, objetos de aprendizaje, docencia síncrona, estaciones de bombeo, hidráulica.

\section{Introducción}

La docencia a distancia basada en herramientas de teleformación a través de Internet es cada día más importante. Además, y cada vez con más facilidades, las herramientas de conexión simultánea, permiten el desarrollo de herramientas de docencia síncrona de forma que los alumnos se encuentran en un espacio virtual con el profesorado con una interacción mucho más directa que los tradicionales cursos a distancia.

En un nuevo marco universitario, se está pasando de un aprendizaje tradicional a un aprendizaje en que el alumno es un sujeto activo, con una mayor implicación, actividad y protagonismo (Pagano, 2007). Para ello las nuevas tecnologías son de gran utilidad, puesto que fomentan un aprendizaje colaborativo y la participación de los estudiantes incluso cuando estos no se encuentran presentes en el aula con el profesor. El uso de herramientas de Internet en la teleformación es una herramienta que viene utilizándose ya desde finales del pasado milenio (Garrison, 1998) y su potencial es enorme. La apertura de estos materiales a foros internacionales enriquecen el contenido y las consultas de los alumnos. Estas experiencias no son nuevas y se encuentran documentadas en la Universidad española desde los primeros años del milenio (Onrubia et al, 2006).

Al hilo de estas experiencias, el trabajo desarrollado por los autores del presente documento se focalizan en la presentación de los resultados que la impartición de forma combinada, a través de docencia síncrona y asíncrona, ha tenido el "Curso Básico Online de Estaciones de Bombeo" realizado en la Universitat Politècnica de València en su primera edición en el curso 2013-2014. 
El curso Básico Online de Estaciones de Bombeo va orientado al conocimiento básico de los elementos que cobran importancia en la gestión de una estación de bombeo como parte de las instalaciones hidráulicas. Se conocerá en esencia los elementos principales de este tipo de instalaciones y las leyes teórico-prácticas que regulan su funcionamiento para una mejor gestión energética y sostenible de las mismas.

\section{Objetivos y metodología del Curso Básico Online de Estaciones de Bombeo}

El objeto de este trabajo es poner en valor la experiencia que los autores han tenido desarrollando un curso específico a distancia, diseñado para profesionales del sector, mediante tecnologías de docencia síncrona. El curso en cuestión ha sido el Curso Básico Online de Estaciones de Bombeo, pensado para profesionales que trabajan en el diseño, proyecto, y operación de redes de distribución de agua potable, agua de riego, o aguas industriales y/o de redes de evacuación de aguas usadas. No obstante, el curso está abierto a todo tipo de participantes, lo que permite comparar su utilidad entre cohortes de difentes procedencia.

El Curso Básico de Estaciones de Bombeo responde a unos objetivos docentes para transmitir a los alumnos, de un lado, los contenidos en relación con la instalación hidráulica y eléctrica de las estaciones de bombeo; y de otro lado, las experiencias en cuanto a los casos de estudio particulares que pueden tener un interés más práctico y aplicado alos profesionales. Estos casos, a través de los foros y los talleres, van desgranando unas habilidades que se espera que los alumnos adquieran más allá de las propias semanas en que se extiende el curso.

El seguimiento del curso se ha realizado en dos fases. Durante la primera semana los alumnos han tenido ocasión de seguir las clases de docencia síncrona a través de la herramienta de Policonecta. De esta forma se ha preparado la información en unidades didácticas de diez minutos, seguidas de turno de preguntas por parte de los alumnos. Este momento ha servido como un foro online en que los alumnos procedentes de la empresa han presentado sus casos y dudas; y los alumnos procedentes del ámbito universitario han compartido las oportunidades que les sugerían cada uno de los tema.

En una segunda fase, el material impreso que los alumnos han recibido y la posible consulta a los objetos de aprendizaje relacionados con cada uno de los puntos de estudio, permite a los alumnos hacer un seguimiento más maduro y con su propio ritmo, del contenido de los temas, dejando siempre abierto un foro para el diálogo entre los componentes del grupo.

Finalmente, el seguimiento del aprendizaje de la materia, se ha conseguido a través de un portafolio de talleres que se han evaluado por parte del profesorado especializado de cada tema. 
La enseñanza síncrona a distancia como método de formación continua a profesionales. La experiencia del "Curso Online de Estaciones de Bombeo"

\section{Desarrollo de la innovación}

Este curso se presenta como una herramienta novedosa en el campo de la formación en ingeniería hidráulica a distancia por las siguientes razones:

- Los alumnos que acceden al curso tienen una formación básica heterogénea y hay que adaptarlo a todos ellos. Además algunos tienen un marcado perfil profesional, lo que hace que la metodología docente deba adaptarse a alumnos con un perfil principiante y con un perfil avanzado. Esto requiere del profesor un conocimiento particular de la materia y la forma de transmitir los contenidos que a su vez, debe adecuarse a la herramienta de comunicación, que es Policonecta (Adobe conect)

- Los conocimientos deben asentarse en los alumnos y evidenciarlo a través de los talleres que componen el portafolio de evaluación. Sin embargo, por el carácter práctico del curso y el perfil de los participantes, los análisis de caso se presentan muchas veces como problemas a abordar durante el desarrollo de las clases. Hay que adaptar los contenidos docentes a los casos que interesan a los alumnos, para que a la vez que se transmiten los conceptos básicos para alumnos menos avanzados, puedan resolverse los casos que interesan a los que lo son más.

- Se utilizan las herramientas de teleformación propias de las Universitat Politécnica de Valencia como son Policonecta, bajo la plataforma Adobe Connect ${ }^{\circledR}$, y POLIFORMA-T, dentro del entorno SAKAI.

La plataforma Policonecta es una herramienta de teleformación que permite realizar conferencias, seminarios, o reuniones a distancia.

La herramienta permite diferentes roles ("Roles existentes en la sala de Polimedia") de tal forma que personas que se encuentran en diferentes lugares pueden ser quienes impartan las conferencias. Existe un rol de anfitrión que ejerce de gestor de la sesión y que, por defecto, actúa de ponente. A la sesión se unen los diferentes participantes a través de un enlace web. El anfitrión puede permitir que cualquier persona conectada a la sesión actúe como "profesor”, lo que le permite que también actúe de ponente.

El resto de miembros de una sesión son alumnos. No son sujetos pasivos, sino que pueden participar planteando preguntas, que pueden ser orales o escritas, a elección del anfitrión. SI el número de alumnos es elevado, para la buena práctica docente, la participación de los alumnos suele ser escrita.

La plataforma POLIFORMA-T (CENTRO DE FORMACIÓN PERMANENTE, 2015) permite a profesores y alumnos interaccionar y compartir contenidos sobre el curso que 
están llevando a cabo. Dispone de una serie de herramientas de teleformación como: repositorio de contenidos, exámenes a distancia, repositorio de tareas (trabajos y casos que los alumnos van realizando y que entregan para su valoración), espacio compartido, foros y chats, etc...

La plataforma es de acceso individual para cada alumno de tal forma que parte de la información allí contenida es exclusiva del alumno.

\section{Resultados del desarrollo de la primera edición}

En la primera edición del curso, el $84 \%$ de los participante han sido profesionales dedicados de una forma u otra a la hidráulica. De entre ellos, un $20 \%$ han sido profesores universitarios, con más interés en la tecnología didáctica que en los propios contenidos tratados.

El alumnado ha seguido de forma asidua la docencia síncrona, participando en su totalidad en todos los momentos de foro abierto. La conexión a las sesiones síncronas ha sido, prácticamente, del 100\% (en un caso hubo una desconexión justificada previamente, precisamente por motivos laborales). A pesar que las sesiones se desarrollaron en horario de tardes, para hacerlo compatible con la asistencia desde países americanos, la mayoría de alumnos, un $84 \%$ han sido asistentes de España.

Las encuestas de valoración del curso por parte de los alumnos han sido positivas, con calificación media de notable. Los alumnos han sido preguntados por aspectos concretos referidos a la teleformación. A la pregunta de "Creo que la formación a distancia es una forma cómoda de reciclarme como profesional?”, la media de las respuestas obtenidas ha sido de 5/5. A la pregunta de: "Opino que el medio utilizado para la formación (Internet) tiene muchas más ventajas que inconvenientes?”, la media de las respuestas ha sido de 5/5.

Los talleres se han presentado y numerosas dudas han sido respondidas durante el período de formación a distancia de los alumnos.

Finalmente, se ha hecho una valoración de los alumnos. Sólo un 35\% de los alumnos se han presentado a la valoración final que les permitía convalidar la formación que créditos de docencia reglada. De ellos un $75 \%$ han sido profesionales, con una califiación media de sobresaliente.

La potencialidad de las herramientas Policonecta y Poliforma-t ha resultado bien demostrada. Ha permitido que conferenciantes de diferentes universidades participen en la impartición del mismo, con tres entidades participantes a la hora de dicción de los contenidos de la docencia síncrona desde sus respectivas sedes en Madrid y Valencia, así como profesionales de la empresa desde Valencia.

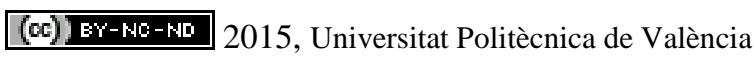

Congreso In-Red (2015) 
La enseñanza síncrona a distancia como método de formación continua a profesionales. La experiencia del "Curso Online de Estaciones de Bombeo"

\section{Conclusiones}

La presente contribución describe la experiencia de los autores en la preparación e impartición del "Curso Online de estaciones de Bombeo" que, desde la plataforma Policonecta de la UPV se ha impartido en el curso pasado en su primera edición y se prepara para el presente curso de nuevo.

La experiencia ha sido altamente satisfactoria, puesto que ha conectado a doce egresados de muy diversas procedencias culturales, locales, regionales y de formación a un único esquema de docencia en el marco de las estaciones de bombeo y la maquinaria hidráulica, con un grupo de profesores procedentes asimismo de la empresa privada, la Universitat Politècnica de València y la Universidad Politécnica de Madrid.

Los alumnos han mostrado un seguimiento importante tanto en las horas de docencia síncrona, desde sus respectivos puestos de trabajo en España e IberoAmérica, como en la preparación y envío de talleres con posterioridad a las clases dictadas. Los foros han sido activos durante todo el tiempo que ha durado la experiencia y en los meses posteriores, lo que da idea del interés que causa el tema propuesto en los alumnos y su entorno práctico.

Para concluir, cabe decir que la experiencia que se describe en esta contribución es altamente positiva y abre el camino a una docencia que combina la teleformación síncrona y asíncrona en un campo en que no existía precendente en la Unviersidad Española como es la maquinaria hidráulica, aplicada a las estaciones de bombeo.

\section{Referencias}

ONRUBIA, J., BUSTOS, A., ENGEL, A. SEGUÉS , T. (2006). Usos de una herramienta de comunicación asíncrona para la innovación docente en contextos universitarios. IV Congreso Internacional de Docencia Universitaria i Innovació. Barcelona. (España).

PAGANO, C.M. (2007). Los tutores en la educación a distancia. Un aporte teórico.» [artículo en línea]. Revista de Universidad y Sociedad del Conocimiento (RUSC). Vol. 4, n. ${ }^{\circ}$ 2. UOC. [Consultada en Abril de 2015 ]. <http://www.uoc.edu/rusc/4/2/dt/esp/pagano.pdf>. ISSN 1698-580X

GARRISON, D. R. (1998). Andragogy, learner-centeredness, and the educational transaction at a distance. Journal of Distance Education, 3. (2), 123-127. 
"Roles existentes en la sala de Polimedia". Centro de Formación Permanente. UPV. $<$ https://media.upv.es/player/?autoplay=true\&id=c5c8ddd7-21b7-8c4b-a89b-872c946fb0c8>

[consulta: mayo de 2015].

CENTRO DE FORMACIÓN PERMANENTE. UPV (2015) "Formación Online Centro de Formación Permanente”. <http://formaciononline.blogs.upv.es/> [consulta: mayo de 2015]

(c)) EY-NC-ND 2015, Universitat Politècnica de València

Congreso In-Red (2015) 